\title{
Evaluation of the tumor volume from surgical specimens after radical prostatectomy and its clinical impact on the prognosis of patients with localized prostate cancer
}

Ye Chang hee, Lee Hak min, Urology, Seoul National University Bundang Hospital, South Korea

Objectives: To predict the prognosis of localized prostate cancer ( $\mathrm{PCa}$ ) patients and evaluate the actual contribution of tumor volume (TV) to the prognosis of $\mathrm{PCa}$.

Patients and Methods: We prospectively measured TV using surgical specimens obtained after radical prostatectomy in 2,316 patients with localized PCa. We also evaluated the clinical effect of TV on postoperative biochemical recurrence using Kaplan-Meier analysis and Cox-proportional model.

Results: The mean prostate volume of the entire cohort was $36.5 \mathrm{cc}$, and the mean TV was 5.9 cc. Significant positive relationships existed between TV and increment of risk group based on the D'Amico criteria and National Comprehensive Cancer Network guidelines $(P<0.001)$. The high TV showed significantly worse pathologic outcomes than the low TV in terms of high rates of extracapsular extension, seminal vesicle invasion, and positive surgical margin $(P<0.05)$. The patients with high TV had significantly shorter biochemical recurrence-free survivals than those with low TV $(P<0.001)$. Finally, based on multivariate Cox-proportional analyses, TV was revealed to be an independent predictor of postoperative biochemical recurrence as both categorical (hazard ratio [HR]: 1.42, 95\% confidence interval [Cl]: 1.13-1.78, $\mathrm{P}=0.003$ ] and continuous variables (HR: $1.04,95 \% \mathrm{Cl}$ : 1.04-1.05, $P<0.001)$.

Conclusions: TV was revealed to be an independent prognostic factor in the postoperative biochemical recurrence. Patients with a high number of positive core and longer tumor length were significantly related to higher TV.

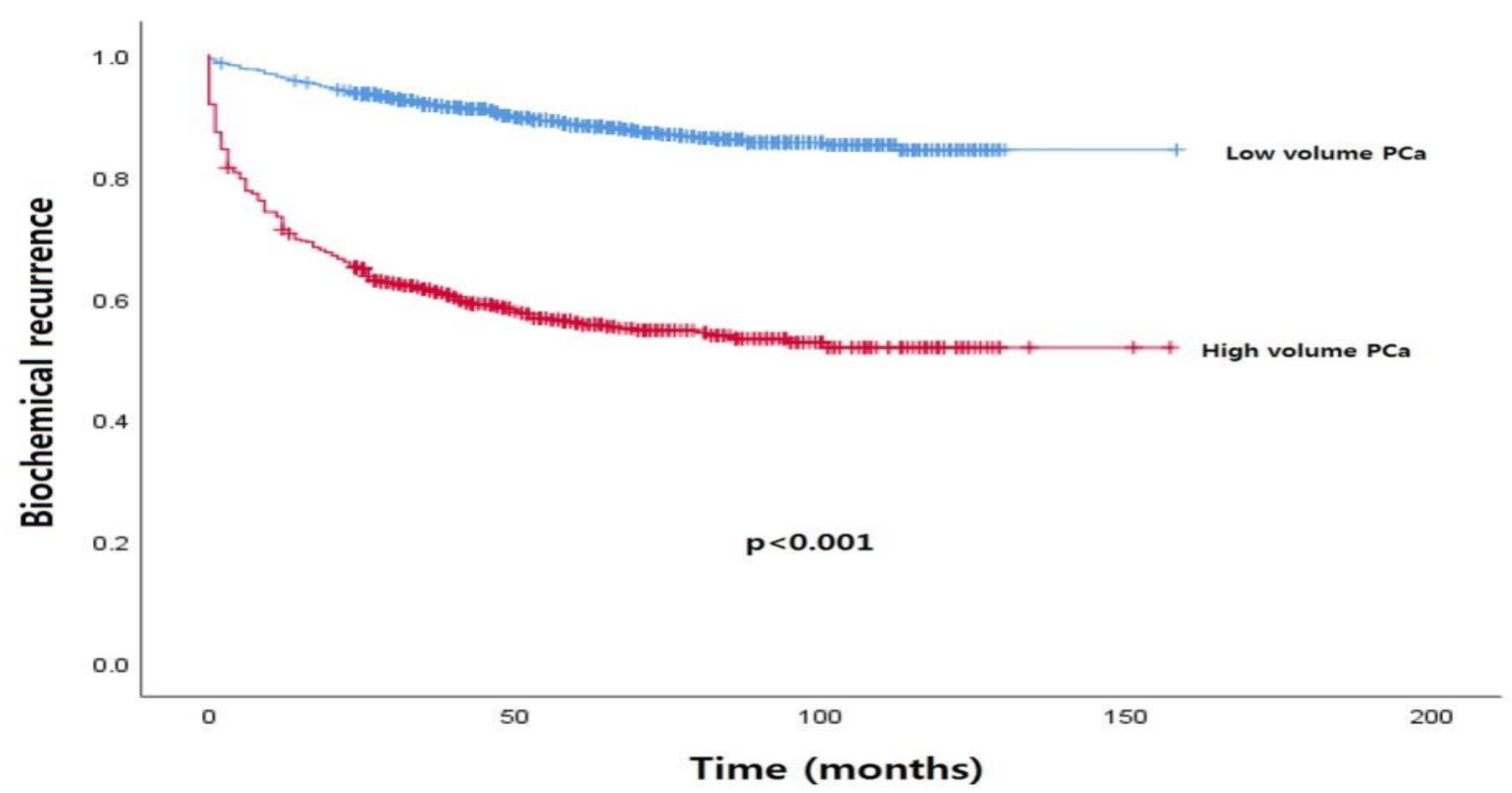

